\title{
AN INTELLIGENT ACTIVE DECISION SUPPORT SYSTEM ARCHITECTURE FOR CRISIS MANAGEMENT
}

\author{
Ahmed S. Salama \\ Computer and Information Systems Department \\ Sadat Academy for Management Sciences (SAMS) \\ Cairo, Egypt \\ Email: \{salama_ahmed@hotmail.com\}
}

\section{ABSTRACT}

Crises (e.g. fires, earthquakes, volcanoes) suddenly occur. Some of them may have previous indications, and some may not. The size and impact of a crisis vary depending on its nature, time, place, potential human casualties, capital losses and environmental circumstances. The proposed architecture in this paper provides an approach for an intelligent active DSS that can predict, detect, and manage crises based on integration of a set of efficient technologies. Thus, this architecture employs the technologies of knowledge based systems, real time data base systems, monitoring systems, and special purpose intelligent agents in a way that ensures the reducing, minimizing or eliminating the consequences of crises when they occur. The main components of the proposed architecture, their functions, and the interaction between them are explained. The architecture presented in this paper can be applied as a generic architecture for any intelligent DSS used for managing crises in a wide range of domains and overcome their bad impacts.

Key Words: crisis management, Active Decision Support Systems, Intelligent Decision Support Systems, Knowledge Based Systems, Intelligent Agents, Monitoring Systems.

\section{Introduction}

Managing a crisis involves various agents/factors that affect the assessment of the crisis, its extent, and how to respond to it. To address issues related to crisis management automation, an intelligent active multiagent DSS integrated architecture is proposed in this paper to assist management teams in dealing with potential crises. The objective is to provide a reliable intelligent integrated DSS architecture for evaluating a crisis, modeling it, and developing an efficient plan for tackling it. Thus, this proposed architecture integrates the data capturing power of continuous monitoring systems, the advantages of using technologies of heterogeneous database systems, the reasoning and inference capability of knowledge based systems, and the intelligent multi-agents.

\section{Background}

Decision making is considered one of the most critical activities done in organizations[10]. To support this complex process for individuals, a variety of independent, standalone information systems called Decision Support Systems (DSSs) have been developed in the two last decades. They are defined as computer-based tools used to support complex decision-making and problem solving [9] .

In the following three sections, several definitions of Decisions Support Systems(DSS), Intelligent Decision 
Support Systems (IDSS) and the importance of intelligent agents based decision support systems have been reviewed.

\subsection{What is a DSS}

There are many definitions of what a DSS is but according to Turban and Aronson [11] the central purpose to a DSS is to support and improve decision making. Little [3] defines DSS as a "model-based set of procedures for processing data and judgments to assist a manager in his decision-making." He argues that to be successful such a system needs to be adaptive, easy to use, robust and complete on important issues. These features are desired but not required in a DSS. Bonczek et al. [1] defines a DSS as a computer-based system consisting of three interacting components a language system, a knowledge system and a problem-processing system. This definition covers both old and new DSS designs, as the problem processing system could be a model-base or an ES or an agent-based system or some other system providing problem manipulation capabilities.

Keen [2] applies the term DSS to situations where a 'final' system can be developed only through an adaptive process of learning and evolution. Thus he defines a DSS as the product of a developmental process involving the builder, the user and the DSS itself combining to evolve into a combined system. For the purpose of this research the definition of a DSS is based on the definitions of Turban and Aronson and Bonczek in that a DSS is a system to support and improve decision making. However, it also must contain the three subsystems that Bonczek describes, that is it must have a database of some kind attached (data subsystem), it must have a mechanism for processing the data (model subsystem) this could be models, rules, agent-based subsystem or some other techniques and it must be capable of receiving and acting on requests from users (user interface subsystem) for example the user might request the detail that allowed a specific recommendation to be formed in order to see for himself if it is justified.

\subsection{Difference Between an IDSS and a DSS}

There are several different definitions of the differences between a DSS and an IDSS. This is due to the fact that there are multiple different kinds of IDSS (or Knowledge- Based
DSS as they are also known), these include those that replace the model base management system with the Expert Systems (ES) or other intelligent decision making functionality, those where the functionality is added to enhance the model- base management system (MBMS) to make it intelligent, and improved user interfaces can be achieved using other parts of artificial intelligence, including natural language processing or similar techniques [11,7].

IDSS also allow for supporting a wider range of decisions including those with uncertainty. So that rather than just making a recommendation it can also give a confidence level that the recommendation is a good one. It can also handle domains where the decision process is more complicated and requires expertise as well as assessment of the impact of the proposed solution [11].

Some other advantages proposed by Marakas [4] gives the advantages of using intelligent components with DSSs as opposed to plain DSSs as increased timeliness in making decisions, improved consistency in decisions, improved explanations and justifications for specific recommendations, improved management of uncertainty, and formalization of organizational knowledge. The most useful of these advantages is the improved explanations and justifications which is an extremely useful feature particularly in fields like medicine, etc where it helps if the real expert can validate the machines reasoning

McGregor [5] uses a Agent-based decision support system that has the intelligence section attached to the model base as opposed to replacing it. This is normally done when the purpose of the intelligence section is to enhance and supplement the models as opposed to replacing them, this can be a very useful method of increasing the accuracy and consistency of decisions. In the context of this research the definition of an IDSS is that the IDSS has an intelligent component, which either replaces or enhances the model subsystem.

\subsection{Intelligent Agents Based Decision Support Systems}

Under conditions associated with real-time decisions, humans may experience stressors such as information overload, time pressure, emotional intensity, and uncertainty. These pressures increase with distributed teams that must 
collaborate to make decisions in rapidly changing environments over networks due to factors such as delays or misunderstandings in communication. Intelligent decision support systems attempt to mitigate these issues by integrating capabilities from the human user and artificial intelligence.

One of the most promising technologies for real applications is intelligent agents. Agents are generally described as software programs that are autonomous, adaptive, proactive, reactive, communicative, cooperative, mobile, goal- driven and persistent in performing their tasks. Using these characteristics, agents and multi-agent teams can be integrated into decision support systems to perform tasks such as monitoring, alerting, learning, and even making decisions. [8]

\section{The Proposed Intelligent Architec- ture}

In the following sections, the main components of the proposed architecture, and their functions are explained. The proposed integrated architecture components and the interactions between them are shown in figure 1 .

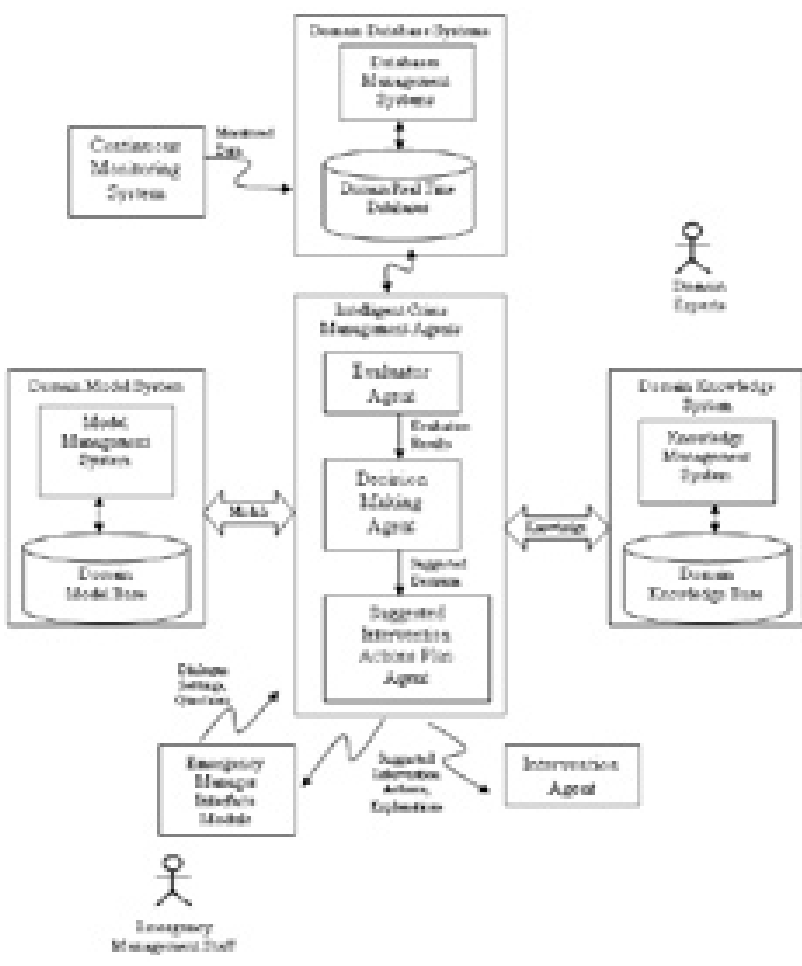

Fig. 1 A Proposed Integrated Intelligent Active DSS Architecture for Crisis Management

\subsection{Continuous Monitoring System}

The continuous monitoring system main goal is to maintain the active property of the intelligent DSS ON. This component main functions are as follows:

a. Keeping track of the domain of interest which is vulnerable for the crisis occurrence or the domain while it is affected by the crisis. (e.g. fire, earthquake, volcano, city, building, important district).

b. Providing the main source of all the real time data that will be stored in the domain miscellaneous real time data bases systems.

c. Providing a continuous feedback about the progress, results and consequences of applying the intervention actions to overcome the crisis, which will be the base for the intelligent DSS to react according to the latest real time changes in the domain.

The continuous monitoring system consists of different types of monitoring technologies varies from very simple to complex ones. Examples of such monitoring technologies:

a. Wired and Wireless Sensor Networks[6].

b. Voice Recognition systems.

c. Satellite systems.

d. Video Recording systems.

e. Pattern Recognition systems.

These networked continuous monitoring systems could be based on internet, extranet, or intranet in transferring their monitoring resulted data to the real time data bases.

\subsection{Domain Database Systems}

The domain Database systems consists of miscellaneous types of database management systems (DBMSs) and databases that could be distributed or centralized according to nature of the crisis domain geography, and management preferences. The used types of database systems could be as follows:

a. Geographical database system (geographical information system) that stores the spatial data in the form of multi layer maps and can perform all the spatial analysis and responds to all the online queries concerning the crisis geographical progress.

b. Image Storage and Management database systems. 
c. Audio and Video management database systems.

d. Numeric and Textual management database systems.

All of these types of database systems could be used or some of them according to the crisis domain nature. The data collected and stored in these different types of database systems are captured by the continuous monitoring systems.

An additional object relational database must be created and managed by an object relational DBMS such as Oracle 10i or SQL Server to store all the resulted and intermediate data that represents the output of running the evaluator agent, decision making agent, and the suggested intervention actions plan agent.

\subsection{Domain Knowledge System}

The domain knowledge based system that consists of the following main components:

a. Knowledge Base that holds all the knowledge elicited from the crisis domain human experts, and also the accumulated knowledge from previous successfully managed similar occurred crisis which will support all the main functions of the evaluator agent, decision making agent, and the suggested intervention actions plan agent. The knowledge could be represented using one or more representation techniques such as production rules, frames, and decision trees. The knowledge base supports all the stages of crisis management which can be determined as predicting, detecting, and preventing or overcome the crisis and minimize or eliminate its bad impacts.

b. Knowledge management system which includes the Inference Engine, the ability to add, retrieve, update, and disseminate through different types of networks the knowledge in the knowledge base. The inference engine guides the reasoning process using the knowledge stored in the knowledge base to support the main functions of the crisis management agents.

All the facts, and data related to the crisis progress and used by the knowledge base system in the reasoning process is stored in the domain database systems.

\subsection{Domain Model System}

The Domain Model System consists of the main following components:

a. Model Base: which consists of all the quantitative, qualitative and descriptive models that supports in the prediction of the crisis progress such as building simulators of fire propagation, and explosion consequences. Also the models supports in the decision making process under certainty, risk (stochastic decision situations), and uncertainty since each model involves all the decision variables, uncontrollable variables, and result variables in the used models. All the values of the models variables, are stored in the real time database.

b. Model management system that enables the activation, selection, and running of the appropriate models in the model base according to the nature of the crisis domain. The selection of the appropriate model(s) can depend on the knowledge in the knowledge base.

\subsection{Intelligent Crisis Management Agents}

The intelligent crisis management agents represent the brain and the most intelligent core in the proposed architecture. In the following subsections, the main three crisis management agents and their functions are explained.

\subsubsection{Evaluator Agent}

The evaluator agent is a software that is responsible for evaluating the domain situation, and answering the following important questions:

a. What is the probability that a crisis is going to take place in the near future?

b. If the crisis occurred, What is the current situation, how is it progressing, and what is the emergency level?

In order that the evaluator agent answers the previous questions, it executes continuously the following algorithm:

a. Retrieve the real data that represents the current situation from the real databases, which are stored by the continuous monitoring system.

b. Run the knowledge management system, to apply the evaluation knowledge in the knowledge base on the real time current domain situation data and pro- 
vides an assessment of the crisis and its impacts, and also applying the knowledge that decides the most appropriate models to run from the models base.

c. Run the appropriate models (quantitative, qualitative, or descriptive models), decided by the domain knowledge system through the Models management system. The execution of models will calculate the values of the risk factors, the decision variables, the uncontrollable variables, and the result variables. In such a way, the crisis propagation and progress will be determined and decided.

d. Use the evaluation knowledge in the knowledge base to evaluate the models results which are stored in the real databases.

e. Send the final evaluation results of the crisis possibility or progress to the decision making agent.

\subsubsection{Decision Making Agent}

The decision making agent is a software that is responsible for determining a set of alternative scenarios to overcome the crisis, evaluating these scenarios, and deciding the most appropriate scenario to follow and apply. The following algorithm is continuously executed:

a. Run the inference engine of the knowledge management system, to apply the knowledge in the knowledge base on the current crisis situation or determine whether the crisis may occur, and generate a set of alternatives based on expertise to overcome the crisis or prevent the crisis from occurring.

b. Run the appropriate models (quantitative, qualitative, or descriptive models) from the models base according to the generated alternatives from the domain knowledge system. Thus a calculated results for each alternative can be determined or a simulation for the outcome of each alternative can be created and tested.

d. Use the knowledge and preferences rules provided by the domain experts in the knowledge base to evaluate the outcomes of each alternative, and choose the best alternative to apply. e. Send the suggested decision to the suggested intervention actions plan agent.

\subsubsection{Suggested Intervention Actions Plan Agent}

The suggested intervention actions plan agent is a software responsible for building a detailed actions plan for the chosen scenario based on the extracted knowledge and the real data available in the databases. This actions plan is transferred to the intervention agent or/ and the emergency manager interface module.

\subsection{Intervention Agent}

The intervention agent is a self automated computerized system that receives the actions plan from the suggested intervention actions plan agent and apply it automatically without any human intervention. This kind of agent is used in certain types of crisis management systems that require quick auto intervention such as fires, earthquakes, and explosions. Usually, the intervention system consists of a set of networked specialized devices (such as sensors, convertors, controllers) controlled by a centralized computer that manage the functions of these devices according to the actions plan to prevent the crisis before occurring or minimize, and end its bad impacts.

\subsection{Emergency Manager Interface Mod- ule}

The emergency manager interface module is the software responsible for enabling the emergency manager to enter his on line questions, queries, settings, preferences and displaying the suggested actions plan to follow in order to overcome the crisis. This module is highly interactive, friendly easy to use graphical user interface, and it is recommended that the dialogue with the emergency manager will be natural language based.

\section{Conclusions and Future Work}

There was always a need for a generic conceptual architecture of crisis management systems. There was not a clear standardized methodology for how to face or predict a crisis no matter its domain. The proposed intelligent active decision support system architecture 
for crisis management in this paper, represents a true trial in providing a generic architecture that can be used for managing crises in a wide range of domains and overcome their bad impacts.

The proposed architecture employs a powerful integration of a set of very effective advanced technologies including miscellaneous real time database systems, knowledge based systems, model based systems, and intelligent agents. The aim of such integration is to provide a robust, reliable, intelligent active DSS that is capable of predicting the crisis before occurrence, and minimizing or eliminating the bad impacts of the crisis if it occurred.

It is recommended in the future to implement this proposed architecture for crisis management in many domains. It is also important to show in our future work, the significance of mobile agents and wireless systems role in managing crises, especially if the crisis was covering a long distance and spreading fast to invade wide areas i.e. districts, cities, and countries.

References

[1] Bonczek, R.H., Holsapple, C. W., and Whinston, A. B., The Evolving roles of Models in Decision Support Systems. Decision Sciences, Vol. 11, Issue 2 - 1980.

[2] Keen, P.G.W., Adaptive Design for Decision Support Systems. Data Base, Vol. 12 Issue 1 and 2 - 1980.

[3] Little, J.D.C., Models and Managers: The Concept of a Decision Calculus. Management Science, Vol. 16 Issue 8 - 1970.

[4] Marakas, G.M., Decision Support Systems in the 21st century, Prentice Hall - 1999.

[5] McGregor, C., and Kumaran, S. An Agent-Based System for Trading Partner Management in B2B eCommerce. in 12th International Workshop on Research Issues in Data Engineering: Engineering e-Commerce/ e-Business Systems (RIDETM02), IEEE - 2002.

[6] Min Chen, Taekyoung Kwon *, Yong Yuan +, and Victor C.M. Leung, Mobile Agent Based Wireless Sensor Networks, JOURNAL OF COMPUTERS, Academy Publisher, VOL. 1, NO. 1, APRIL 2006.

[7] Sauter, V., Decision Support Systems: An Apllied
Managerial Approach, John Wiley \& Sons, Inc. - 1997.

[8] Phillips-Wren, G. , Intelligent decision support to assist real-time collaboration, Collaborative Technologies and Systems, 2008. CTS 2008. International Symposium on, Vol. 3, pp 375-375 - May 2008.

[9] Shim J.P., et al., Past, present and future of decision support technology. Decision Support Systems Vol 33, - pp111-126 - 2002.

[10] Simon, H.A., Administrative behaviour: a study of Decision-Making process in Administrative Organizations. New York, Free Press - 1997.

[11] Turban, E., and Aronson, J. E., Decision Support Systems and Intelligent Systems, Prentice Hall -7th ed. -2004.

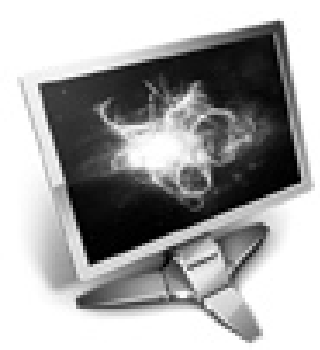

